\title{
Meta
}

Journal des traducteurs

Translators' Journal

\section{Saez Hermosilla, Teodoro (1994) :El sentido de la traducción : reflexión y crítica, León, Universidad, Secretariado de Publicaciones ; Salamanca, Universidad, HOp.}

\section{Georges L. Bastin}

Volume 41, numéro 1, mars 1996

Le(s) processus de traduction / Translation Process(es)

URI : https://id.erudit.org/iderudit/001994ar

DOI : https://doi.org/10.7202/001994ar

Aller au sommaire du numéro

Éditeur(s)

Les Presses de l'Université de Montréal

ISSN

0026-0452 (imprimé)

1492-1421 (numérique)

Découvrir la revue

Citer ce compte rendu

Bastin, G. L. (1996). Compte rendu de [Saez Hermosilla, Teodoro (1994) : $E l$ sentido de la traducción : reflexión y crítica, León, Universidad, Secretariado de Publicaciones ; Salamanca, Universidad, HOp.] Meta, 41(1), 179-181.

https://doi.org/10.7202/001994ar d'utilisation que vous pouvez consulter en ligne.

https://apropos.erudit.org/fr/usagers/politique-dutilisation/ 
- SAEZ HERMOSILLA, Teodoro (1994) : El sentido de la traducción : reflexión y crítica, León, Universidad, Secretariado de Publicaciones; Salamanca, Universidad, $110 \mathrm{p}$.

Voilà un essai comme on n'a plus l'habitude d'en lire. Écrit à la première personne, il nous livre une réflexion théorique bien personnelle d'un auteur qui décrit, explique et choisit, un auteur qui a le mérite de critiquer positivement les œuvres qu'il choisit mais qui sait prendre position. Son moindre crédit n'est pas d'avoir publié ces réflexions et critiques en espagnol, langue dans laquelle on trouve encore trop peu d'ouvrages, en dépit du nombre croissant d'experts (hispanophones).

Sáez Hermosilla commence par revisiter quelques problèmes théoriques fondamentaux liés à la traduction (comme les universaux du langage, la non-isomorphie langue/pensée, la fidélité) pour ensuite centrer son attention sur les différences entre traduction écrite et traduction orale, la théorie du sens, la traduction artistique, la théorie des espaces perceptuels linguistiques et la translémique. Chacune de ces approches théoriques fait l'objet d'une critique à la fois rigoureuse et subjective. Le livre conclut sur une proposition de théorie du langage en tant qu'action sociale.

Dès l'avant-propos, l'auteur précise ses fins :

«corriger et préciser... une hypothèse théorique qui m'a séduit depuis le début... une intuition heuristique qui semble raisonnable et investie de bon sens et d'avenir... la Théorie du sens ou Théorie de l'Ecole de Paris» (p. 10) (notre traduction).

Parmi les problèmes théoriques fondamentaux revisités par Sáez Hermosilla, le premier est celui des universaux du langage dont il est convaincu qu'ils ne peuvent être trouvés dans les systèmes linguistiques mais plutôt dans l'univers de la parole et de la communication, celle-ci «fonctionnant» au-delà et en dépit des langues. Il confirme notamment la non-isomorphie langue/pensée malgré leur indépendance : l'idée même de traductibilité en dépend... Quant à la fidélité, trois dimensions interviennent dans l'analyse : la subjectivité du traducteur, l'historicité et la fonctionnalité, reprenant ainsi la contribution précieuse d'une collègue hispanophone, Amparo Hurtado Albir ${ }^{1}$.

La théorie du sens, nous dit l'auteur après l'avoir décrite et raisonnée, est une «théorie valable», «l'une des principales réussites et originalités dans le traitement de la question millénaire du langage et de la traduction» (p. 41). Sans exposer de façon systématique les postulats de cette théorie, on nous rappelle quand même que «le langage dépend,.... des compléments cognitifs non verbaux...» (p. 38) et que la traduction est un «acte interprétatif... /qui/ agglutine au signifié purement linguistique, les intentions du 
dire, des connaissances, des croyances et des sous-entendus, la prise en compte d'une circonstance et d'un contexte, des stratégies à l'endroit du destinataire et un modèle ou genre de discours» (p.38). De même, on insiste sur le processus de la traduction et l'importance de la phase de conceptualisation, judicieusement assimilée au «débat préverbal» chez le locuteur qui, avant de prendre la parole, «s'imagine» son interlocuteur.

Cette adhésion de l'auteur aux thèses de l'École de Paris n'exclut cependant pas un certain nombre de critiques. Il estime en effet que cette théorie n'a pas abouti à une «méthode cohérente et globale, ni même dans le cas de Delisle...» (p. 44). Sáez Hermosilla, surtout, ne considère pas pertinente, dans le cas de la traduction écrite, cette «tendance radicale à mener l'original au destinataire en employant la déviation délibérée de la littéralité» qui représente, pour lui, «une option très discutable». Les partisans de cette démarche «ne peuvent se limiter... à reformuler le vouloir-dire de l'original», mais doivent recréer tout ce qui est «essentiel» aux textes : mode d'expression et sentiments. Une traduction interprétative «ne s'applique guère qu'à la pratique de la traduction orale» (p. 46). L'auteur en arrive ainsi à soutenir, pour les textes littéraires - qui d'ailleurs semblent le préoccuper de manière prioritaire -, l'idée de la traduction sémantique selon Newmark.

Autre critique : la théorie du sens se fonde davantage sur le processus que sur les résultats formels ; or, affirme Sáez Hermosilla «le phénomène de la traduction a le droit d'être envisagé aussi comme une comparaison de résultats» (p. 48). Pour l'ESIT, la confrontation ne porte que sur les contenus et ne cherche pas le moyen terme d'équilibre formel, les compromis structurels et stylistiques. Il critique le fait de privilégier à l'extrême la reformulation selon la norme de la langue cible; pour l'auteur, il devrait être possible de «réécrire un texte, pas nécessairement pragmatique, en combinant la clarté et l'exactitude des contenus idéiques et en conservant un certain exotisme dérivé, non des structures linguistiques mais du style de l'original comme type de discours et comme modèle d'expression» (p. 48).

En ce qui concerne la traduction artistique, question omniprésente dans l'ouvrage, l'auteur se limite à poser la difficulté d'UNE théorie de la traduction artistique dans quatre domaines d'application: poésie, conte, roman et théâtre. S'il affirme que la traduction artistique est possible, de même qu'une certaine théorisation, il ne nie pas que chaque œuvre appelle une démarche traductologique spécifique. Il reconnaît également que, dans tous les domaines de la traduction - à plus forte raison en poésie -, une perte semble inévitable du fait de contraintes catégorielles les plus diverses selon les domaines.

La théorie des espaces perceptuels linguistiques (EPL) de Mariano García Landa occupe une place de choix dans cet essai, d'autant que l'auteur aurait, pendant plusieurs années, collaboré à sa conception et mise au point aux côtés de García Landa dont la thèse de doctorat, la première en interprétation à l'ESIT en 1978, s'intitulait : Les déviations délibérées de la littéralité en interprétation de conférence. Cette théorie, qui, selon Sácz Hermosilla, prolonge et enrichit celle du sens, «se veut, à la fois, une théorie de la parole et une théorie du 'meaning'» (p. 57). Elle postule que, quand nous prenons la parole, nous produisons des percepts linguistiques; ceux-ci construisent la réalité sociale des hommes qui se comprennent grâce aux 'actes de langage'. Sur cette base, les concepteurs exposent une série d'axiomes et de principes (de moralité, totalité, quotidienneté, etc.) permettant de formuler 17 définitions qui constituent un modèle descriptif des faits de parole, lesquelles, à leur tour, donnent lieu à deux formules (celle du discours de l'orateur et celle de la traduction). Viennent ensuite plusieurs théorèmes (équivalence sémantique / équivalence conceptuelle et contextuelle, la litéralité et ses déviations, espace du signe / espace du sens, etc.). Bref un corps théorique complexe et dense auquel s'intègrent les apports des disciplines les plus diverses depuis la philosophie du langage 
et la psychologie cognitive, en passant par l'herméneutique et la pragmatique, jusqu'à la théorie des actes de langage...

Triste ou déçu de ne pas voir culminer ce développement théorique avec son collaborateur, en tout cas insatisfait de la tournure des événements, Teodoro Sáez Hermosilla signale un certain nombre de lacunes et de déviations de cette élaboration théorique. La plus grave de celles-ci est de négliger l'apport récent de disciplines «linguistiques», en particulier de la sémiotique, la stylistique et la rhétorique, notamment de travaux sur la prosodie et le rythme des discours, ainsi que sur l'analyse littéraire. Pour se justifier, il revient d'ailleurs sur certains littéralistes comme Ortega et Berman, et fait appel à des auteurs comme Meschonnic et Etkind, ou à ses compatriotes espagnols Núnez Ladevéze, Pozuelo Yvancos, Lozano, entre autres.

Le dernier courant théorique présenté et critiqué par l'auteur est celui de la translémique, né à l'École de León et dont les représentants sont Julio-César Santoyo et Rosa Rabadán ${ }^{2}$. Le traitement de l'équivalence translémique est certes une démarche qui éclaire sous un jour nouveau tant les phénomènes que les processus de traduction. Inspirée de la linguistique textuelle et de la littérature comparée, cette théorie s'alimente également des travaux de Toury et de Even-Zohar. Pour la présenter, Sáez Hermosilla choisit de la comparer à L'analyse $d u$ discours... de Delisle. Sans aucun doute une erreur méthodologique, mais qui met en lumière certaines coïncidences et surtout offre l'occasion d'une note en bas de page (30. pp. 80-81) regrettant l'imperméabilité des «Écoles» et le manque de connaissance réciproque...

En conclusion, Teodoro Sáez Hermosilla, avec beaucoup d'éclectisme et quelques contradictions..., prêche pour une Théorie générale du texte ouvrant la voie à une théorie de la parole en tant qu'action sociale. C'est là aussi le but recherché par García Landa. L'expérience de l'auteur avec ce dernier semble en tout cas la cause de l'amertume que l'on retrouve tout au long de l'essai, et qui en fait un essai «introspectif». L'ouvrage donne parfois même l'impression d'un règlement de compte entre les deux cochercheurs. Quoi qu'il en soit, le résultat est là : une somme de réflexions et de critiques, quelques propositions aussi, et de nombreuses pistes de recherche qu'il n'est pas déconseillé de lire attentivement.

\section{Notes}

1. Hurtado Albir, Amparo (1990): La notion de fidélité en traduction, Collection «Traductologie», $\mathrm{n}^{\circledR} 5$, Paris, Didier Érudition, $236 \mathrm{p}$.

2. Voir le compte rendu de Rabadan, Rosa (1991) par Clara Foz dans Meta, 38-3, 1993, pp. 556-558. 